\title{
When novel becomes familiar
}

Novel sensory stimuli elicit adaptive behavioural responses such as alerting the animal to a potentially salient stimulus. The neural circuits that mediate the response to novelty need to respond generally to novelty (regardless of salience) and become suppressed as the stimulus becomes familiar, but these circuits are not well understood. Hattori et al. now identify an olfactory circuit in Drosophila melanogaster that encodes the transition of an olfactory stimulus from being novel to being familiar.

In D. melanogaster, odours are detected by olfactory sensory neurons that activate Kenyon cells (KCs) of the mushroom body (MB), which in turn synapse onto MB output neurons (MBONs) that influence fly behaviour. Importantly, this KC-MBON synapse is modulated by dopaminergic neurons (DANs).

The authors exposed flies to repeated exposure to a novel odour. Calcium transients in different $\mathrm{MB}$ compartments were monitored using the genetically encoded calcium indicator GCaMP6f. Hattori et al. found that the response of MBONs in the $\alpha^{\prime} 3$ compartment showed repetition suppression - that is, it was strong when an odour was novel but diminished rapidly on subsequent exposures. This suppression was retained 20 minutes after odour exposure but lost after 1 hour. These findings indicate that $\alpha^{\prime} 3 \mathrm{MBONs}$ encode novelty of odours and the transition to familiarity.

The transition from novelty to familiarity reflects a form of memory, which in the fly involves dopaminergic modulation of the KC-MBON synapse. The DAN innervating the $\alpha^{\prime} 3$ compartment is the PPL1- $\alpha^{\prime} 3$, and, unlike other DANs, this neuron is activated by odour. Selective silencing of the PPL1- $\alpha^{\prime} 3$ markedly reduced repetition suppression in $\alpha^{\prime} 3$ MBONs. Moreover, RNA interference-mediated knockdown of the dopamine (DA) receptor DAMB selectively in MBONs also attenuated repetition suppression. Together, these data suggest that repetition suppression at the KC-MBON synapse requires release of DA from PPL1- $\alpha^{\prime} 3$ that acts on DAMB receptors on MBONs.

Next, the authors investigated the memory decay underlying loss of repetition suppression that occurs 1 hour after repeated odour exposure. It has previously been shown that coincident $\mathrm{KC}$ activation and DA release within $\mathrm{MB}$ compartments can lead to KC-MBON synaptic depression, whereas DA release without coincident $\mathrm{KC}$ activation can lead to synaptic facilitation. The authors found that, after repetition suppression to one odour, exposure to different novel odours, which leads to DA release without activation of KCs encoding the familiar odour, triggered recovery of responses to the familiar odour. Thus, this bidirectional DA modulation protects the compartment from memory saturation.

Finally, Hattori et al. investigated the role of this circuit in fly behaviour. The authors developed a behavioural assay in which the effects of odour presentation on grooming behaviour could be monitored. Exposure to a novel odour elicited an alerting response that interrupted grooming in around half the flies tested, and this response rapidly diminished on subsequent exposure to the same odour. Novel odours presented after silencing of MBONs disrupted grooming in only $10 \%$ of flies, similar to controls. Together, these findings indicate that, in D. melanogaster, the alerting response to novel odours is dependent on activation of KC-MBON synapses in the $\alpha^{\prime} 3 \mathrm{MB}$ compartment and that familiarity is associated with the suppression of these synapses by DA release from PPL1- $\alpha^{\prime} 3$ neurons.

Sian Lewis

\section{ORIGINAL ARTICLE Hattori, D. et al.}

Representations of novelty and familiarity in a mushroom body compartment. Cell 169, 956-969. e17 (2017)

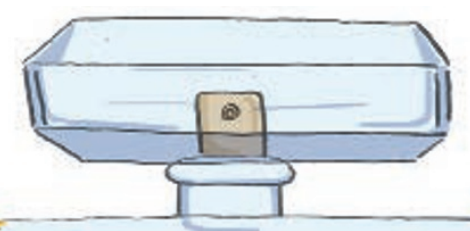

$\alpha^{\prime} 3$ MBONs encode novelty of odours and the transition to familiarity 